\title{
Increased Serum Elabela Level Related to NT-proBNP in Patients with Heart Failure
}

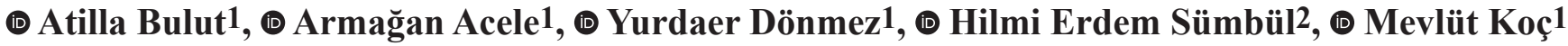

1University of Health Sciences, Adana Health Practice and Research Center, Department of Cardiology, Adana, Turkey

2University of Health Sciences, Adana Health Practice and Research Center, Department of Internal Medicine, Adana, Turkey

\begin{abstract}
Objectives: Apelin levels have been shown to be increased in heart failure with reduced ejection fraction (HFrEF). However, the Elabela level in this patient group and its relationship with laboratory parameters are not clearly known. The aim of this study was to investigate the change in serum Elabela level in patients with HFrEF and its relationship with other clinical, laboratory and echocardiographic parameters.

Materials and Methods: The study included 89 patients with HFrEF and 73 age-sex-matched healthy controls. Serum Elabela level was measured in addition to routine anamnesis, physical examination, laboratory and echocardiography examinations.
\end{abstract}

Results: In patients with HFrEF; aspartate aminotransferase (AST), alanine aminotransferase (ALT), high-sensitivity C-reactive protein (hs-CRP), N-terminal pro B-type natriuretic peptide (NT-proBNP), and Elabela levels were significantly higher than those in healthy controls. Serum Elabela level was found to be positively correlated with blood urea nitrogen, AST, ALT, NT-proBNP, hs-CRP levels, left ventricular (LV) and left atrium (LA) diameters and volumes but negatively correlated with LV ejection fraction (LVEF). It was found that these parameters were only closely related to NT-proBNP, LVEF and LA end-diastolic diameter.

Conclusion: The serum Elabela level in patients with HFrEF is significantly increased, and this is closely related to NT-proBNP, LA end-diastolic diameter and LVEF. Increased Elabela value in patients with HFrEF is closely related to NYHA class-III patients.

Keywords: Elabela, heart failure with reduced ejection fraction, NT-proBNP

Address for Correspondence: Yurdaer Dönmez, University of Health Sciences, Adana Health Practice and Research Center, Department of Cardiology, Adana, Turkey

Phone: +90 5332567896 e-mail: yurdaerd@gmail.com ORCID: orcid.org/0000-0003-4745-7801

Received: 24.11.2019 Accepted: 04.02.2020

Cite this article as: Bulut A, Acele A, Dönmez Y, Sümbül HE, Koç M. Increased Serum Elabela Level Related to NT-proBNP in

Patients with Heart Failure. EJCM 2020;8(1):1-9.

DOI: 10.32596/ejcm.galenos.2020.11.059

${ }^{\circ}$ Copyright 2020 by Heart and Health Foundation of Turkey (TÜSAV) / E Journal of Cardiovascular Medicine published by Galenos Publishing House. 


\section{Introduction}

Heart failure with reduced ejection fraction (HFrEF) is a disease with poor prognosis and is defined as a left ventricular (LV) ejection fraction $<40 \%$ in the last guideline for heart failure $(\mathrm{HF})^{(1)}$. Biochemical parameters and echocardiography are used in the follow-up and treatment of patients with HF, including physical examination, posterior-anterior chest X-ray, electrocardiography, and natriuretic peptide ${ }^{(1)}$.

Many systems are initially activated to compensate for the current situation and to increase cardiac output in patients with HF. Renin-angiotensin-aldosterone system (RAA), sympathetic nervous system (SNS) and natriuretic peptide system are well-known inavestigated systems. These systems are interrelated and are often activated for the purpose of protecting the cardiovascular condition and can thus be used in the diagnosis and treatment of cardiovascular system diseases. Especially RAA system and SNS have long-term negative effects. Apelinergic system is an important peptide family in patients with HF, but not well known as other systems. It has been reported that this system plays an important role in cardiovascular system modulation and has cardioprotective effects via Apelin's peptide and APJ receptor ${ }^{(2-4)}$. Elabela and Apelin antagonize the RAA system in patients with HF, reduce remodeling, increase myocardial vascularity, cause peripheral vasodilation, increase cardiac output, and are therefore considered to play a very important role in preventing cardiovascular disease and slowing disease progression in patients with $\mathrm{HF}^{(2,5-12)}$. To the best our knowledge, there is an increase in Apelin levels in patients with $\mathrm{HFrEF}^{(13)}$. However, the relationship between the Elabela level and the echocardiography and laboratory parameters, which are important in patient follow-up and prognosis, are not clear.

Therefore, we aimed to investigate the changes in the Elabela level in patients with HFrEF and whether these peptides are related to natriuretic peptide and echocardiographic parameters.

\section{Materials and Methods}

\section{Study Population}

In this cross-sectional study, we included 89 patients who were admitted to our hospital's cardiology clinic, who had HFrEF $(\mathrm{EF} \leq \% 40)$, and who were treated according to the New York Heart Association (NYHA) I-II-III class. As for the control group, age and sexmatched adults without any cardiovascular risk factors and active diseases were enrolled to the study. The study was conducted between January and September 2019, and subjects were not included in a prospective way. All patients included in the study had chronic HFrEF. NYHA classifications were evaluated by two cardiologists. In case of a discrepancy in diagnosis, the opinion of another cardiologist was taken. Those with acute coronary syndrome, NYHA class IV, with a history of known acute or chronic liver disease, severe renal failure (eGFR $<30$ $\mathrm{mL} / \mathrm{kg} / 1.73 \mathrm{~m}^{2}$ ), the presence of Hepatitis B or C, regular alcohol use ( $>20 \mathrm{gr} /$ day) or alcohol addiction, severe heart valve disease, portal hypertension, inflammatory diseases, hematological diseases, active thyroid disease, cancer and/or pregnancy suspicion, LVEF $\geq 40 \%$ and patients not willing to participate in the study were excluded from the study. The study was conducted according to the recommendations of the Declaration of Helsinki and Çukurova University Faculty of Medicine, Ethical Committee of Non-invasive Clinical Research approved the protocol (decision no: 30 date: 05.10.2018). Consent forms were explained in detail to all patients and patients were included in the study after written informed consent was obtained.

In all patients, detailed medical history was taken, and physical examination was performed. Subsequently, demographic characteristics of all groups were questioned for age, gender, hypertension, Diabetes Mellitus, active smoking and hyperlipidemia. Pulse rate, systolic blood pressure, and diastolic blood pressure were recorded. Body mass index was calculated by measuring weight and height. 


\section{Laboratory Parameters}

Blood samples were taken from an antecubital vein after the patients rested for 20 minutes in the supine position. Blood samples were collected in tubes containing ethylenediaminetetraacetic acid. Complete blood count was performed. The samples were spun at 3,000 rpm for 10 minutes at $0{ }^{\circ} \mathrm{C}$. At the beginning of the study, glucose, blood urea nitrogen, creatinine, total cholesterol, high-density lipoprotein cholesterol, low-density lipoprotein cholesterol, and triglycerides were measured using standard automated laboratory methods (Abbott Aeroset, MN, USA) and using appropriate commercial kits (Abbott). Serum aspartate aminotransferase (AST), alanine aminotransferase (ALT), uric aside, high-sensitive C-reactive protein (hs-CRP) and $\mathrm{N}$-terminal pro-brain natriuretic peptide (NT-proBNP) levels were also measured using an automated chemistry analyzer (Abbott Aeroset, MN, USA) with appropriate commercial kits (Abbott). High-sensitive troponin I was measured with UniCell DXI-Son-2016 autoanalyser (Beckman Coulter; USA).

Serum Elabela levels were determined using commercial kits (Sunred Biological Technology, Shanghai, China). Elabela-32 isoform was measured. The kit used a double-antibody sandwich enzyme-linked immunosorbent assay (ELISA) to assay the level of Elabela in samples. According to the manufacturer; this assay has inter-assay coefficients of variation less than $12 \%$ and intra-assay coefficients of variation of less than $10 \%$. All of the above tests were performed from blood samples, which were taken at the $24^{\text {th }}$ hour of hospital admission.

\section{Echocardiographic Evaluation}

Echocardiography examinations were performed on EPIQ 7 (Philips Healthcare, Andover, Massachusetts, USA). Images were taken according to the guidelines of the American Echocardiography Society. When the patients were monitored and left-sided, a standard parasternal long and short axis was obtained, as well as apical 5, 4 and 2 space chambers and at least three consecutive cycles ${ }^{(14)}$.
Parasternal long-axis M-mode examinations revealed LV and left atrial (LA) systolic and diastolic diameters. The LVEF was calculated by the modified Simpson method from apical four and two-space chambers ${ }^{(15)}$. Continuous variables that showed normal distribution were compared using the Student's t-test and ANOVA, whereas the MannWhitney U test and Kruskal-Wallis test were used for the samples without normal distribution.

\section{Statistical Analysis}

All analyses were performed using SPSS 22,0 (Chicago, IL, USA) statistical software package program. The distribution of continuous variables was assessed by the Kolmogorov-Smirnov test. Continuous variables ingroup data were expressed as mean \pm standard deviation. Categorical variables were expressed by number and percentage. Continuous variables that showed normal distribution were compared using the Student's t-test and ANOVA, whereas the Mann-Whitney U test and KruskalWallis test were used to compare differences between two independent groups when the dependent variable was either ordinal or continuous, but not normally distributed. Chi-square $(\chi 2)$ test was used to compare categorical variables. The kappa coefficient was used to examine the interobserver variability of the all-echocardiographic measurements. Pearson and Spearman correlation analysis evaluated the existence of a relationship between countable parameters. In the univariate analysis, statistically significant parameters related to serum Elabela level were included in the multivariate model and multivariate linear regression analysis was performed. A logistic regression analysis was performed to determine the independent markers among patients with advanced class HF (NYHA class III). Statistical significance was accepted as $p<0.05$.

\section{Results}

Serum Elabela level was measured from all patients included in the study. The study population was divided into two groups as patients with $\mathrm{HFrEF}$ (55 men, 34 women; and mean age: $52.8 \pm 12.6$ years) and healthy controls (40 men, 33 women; and mean age: 50.1 110.1 
years), and all parameters were compared. There were 62 patients with ischemic cardiomyopathy and 27 patients with non-ischemic dilated cardiomyopathy in HFrEF group. Cohen kappa values that evaluated interobserver and intraobserver variability were over $90 \%$ for all echocardiographic parameters.

When the demographic findings between patients with HFrEF and healthy control groups were compared, it was found that all parameters were similar between the two groups (Table 1). According to the laboratory data, in patients with HFrEF, serum ALT, hs-CRP, NT-proBNP and Elabela levels were significantly higher than those in healthy controls (Table 1). Echocardiography parameters including LA end-diastolic diameter, LV systolic and enddiastolic diameter and volume were significantly higher in patients with HFrEF, whereas LVEF was significantly lower in patients with HFrEF (Table 2).

The parameters that are significantly associated with serum Elabela level in univariate analysis are shown in Table 3. Serum Elabela level was found to be positively

Table 1. Clinic, demographic, laboratory and medical treatment findings according to study groups

\begin{tabular}{|c|c|c|c|}
\hline Variable & $\begin{array}{l}\text { HFrEF patients } \\
(n=89)\end{array}$ & $\begin{array}{l}\text { Controls } \\
(n=73)\end{array}$ & $\mathbf{p}$ \\
\hline Age (year) & $52.8 \pm 12.6$ & $50.1 \pm 10.1$ & 0.131 \\
\hline Gender (male/female) & $55 / 34$ & $40 / 33$ & 0.424 \\
\hline Hypertension, n (\%) & $35(39 \%)$ & - & - \\
\hline Diabetes Mellitus, $\mathbf{n}(\%)$ & $12(14 \%)$ & - & - \\
\hline Current smoker, n (\%) & $52(58 \%)$ & - & - \\
\hline Systolic blood pressure (mmHg) & $118 \pm 14$ & $115 \pm 14$ & 0.211 \\
\hline Diastolic blood pressure (mmHg) & $76 \pm 8.9$ & $77 \pm 9.2$ & 0.665 \\
\hline Pulse (bpm) & $80 \pm 13$ & $76 \pm 11$ & 0.235 \\
\hline Body mass index $\left(\mathrm{kg} / \mathrm{m}^{2}\right)$ & $26.2 \pm 3.8$ & $26.4 \pm 3.2$ & 0.855 \\
\hline High density lipoprotein cholesterol (mg/dL) & $55 \pm 16$ & $52 \pm 12$ & 0.857 \\
\hline Triglycerides (mg/dL) & $121 \pm 40$ & $111 \pm 44$ & 0.112 \\
\hline Aspartate aminotransferase (u/L) & $24.2 \pm 12.5$ & $18.2 \pm 6.1$ & $<0.001$ \\
\hline Alanine aminotransferase (u/L) & $33.1 \pm 37.5$ & $20.8 \pm 13.6$ & 0.005 \\
\hline Blood urea nitrogen $(\mathrm{mg} / \mathrm{dL})$ & $30.7 \pm 6.26$ & $29.1 \pm 6.03$ & 0.082 \\
\hline Creatinine (mg/dL) & $0.75 \pm 0.33$ & $0.70 \pm 0.25$ & 0.141 \\
\hline Uric aside & $6.23 \pm 1.82$ & $5.77 \pm 1.55$ & 0.088 \\
\hline High- sensitive C-reactive protein (mg/dL) & $0.49 \pm 0.15$ & $0.36 \pm 0.24$ & $<0.001$ \\
\hline Spironolactone, n (\%) & $44(49 \%)$ & - & - \\
\hline
\end{tabular}


correlated with blood urea nitrogen, AST, ALT, NTproBNP, hsCRP levels, LA and LA diameters and volumes, and negatively correlated with LVEF in univariate analysis $(p<0.05$, for each one). Linear regression analysis was performed to find the closest relationship between serum Elabela level and these parameters. As a result of this analysis, only NT-proBNP, LVEF and LA end-diastolic diameter were found to be closely related to serum Elabela level ( $\mathrm{p}=0.002$ vs $\beta=0.253, \mathrm{p}=0.001$ vs $\beta=0.267$ and $p<0.001$ vs -0.617 , respectively) (Table $3)$. The relationship between Elabela and NT-proBNP is shown in Figure 1.

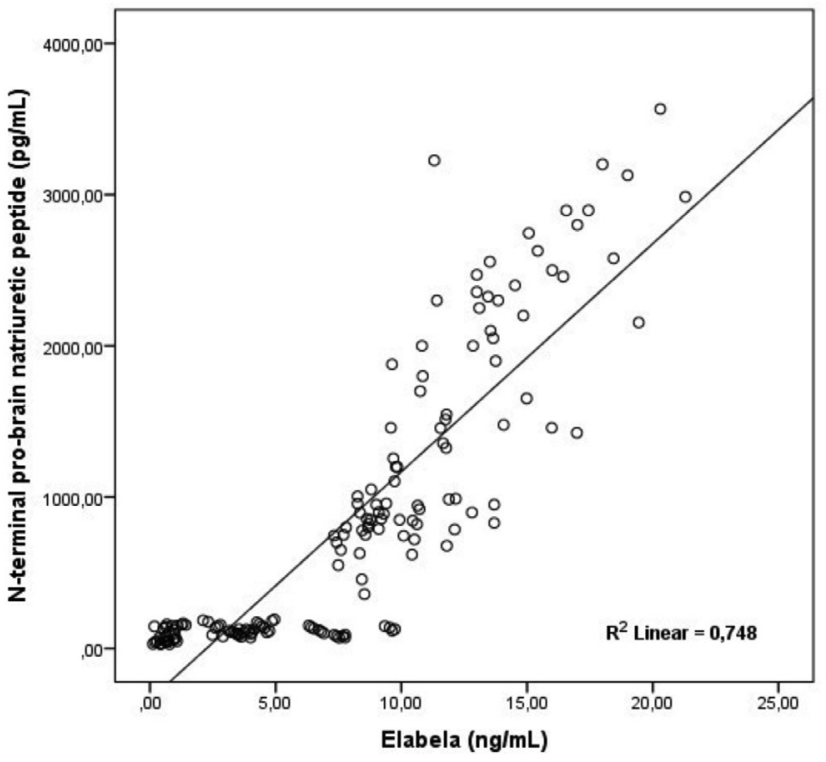

Figure 1. Simple Scatter/Dot graph shows a close relationship between NT-proBNP and serum Elabela

NT-proBNP: N-terminal brain natriuretic peptide
When the clinic, demographic, laboratory, medical treatment and echocardiographic parameters were examined according to the NYHA class, it was found that NT-proBNP and Elabela levels, LA end-diastolic diameter, LV end-diastolic diameter, and volume were the highest in NYHA class III and significantly higher in the NYHA class III than in the NYHA class I $(p<0.05$ and Table 4). An increase in serum Elabela values according to NYHA classes is shown in Figure 2. LVEF was the lowest in NYHA class III and significantly lower in the NYHA class III than in the NYHA class I ( $<<0.05$ and Table 4). It was found that all other clinic, demographic, laboratory

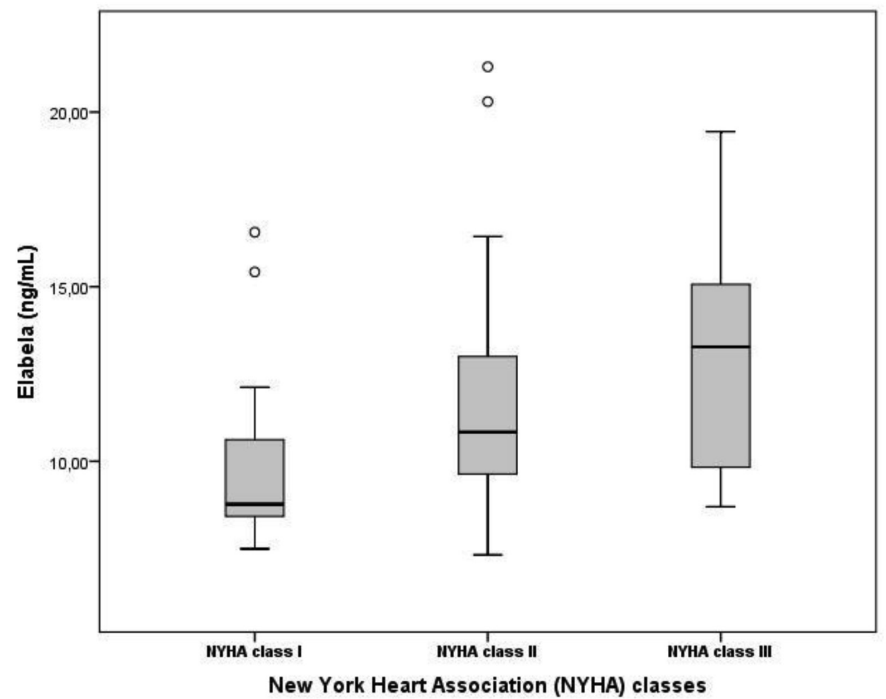

Figure 2. The Boxplot graph shows that serum Elabela values were increased according to NYHA class I to III and highest in NYHA class III

NYHA: New York Heart Association

Table 2. Echocardiographic findings according to study groups

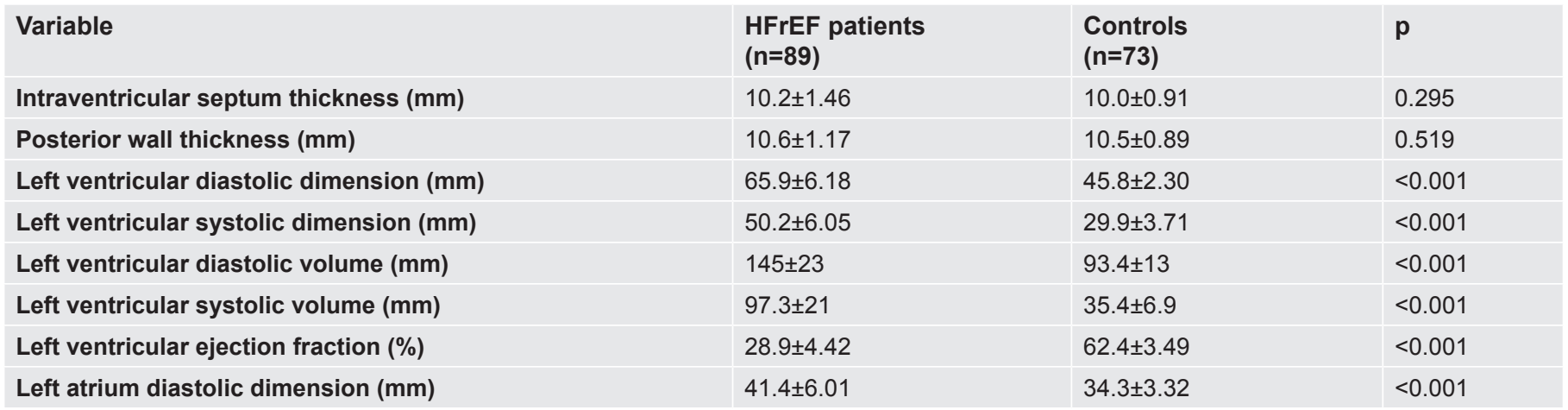


and medical treatment findings were similar between the NYHA groups.

When patients with HFrEF were separated into advanced class HF (NYHA class III) and non-advanced class HF (NYHA class I-II), the Elabela levels were found to be $10.9 \pm 3.07 \mathrm{ng} / \mathrm{mL}$ and $13.1 \pm 3.26 \mathrm{ng} / \mathrm{mL}$, respectively $(\mathrm{p}=0.002)$. When logistic regression analysis was performed to determine for class III patients, it was found that Elabela and LVEF independently determined the presence of having class III disease [Odds ratio (OR): $1.317,95 \%$ confidence interval $(\mathrm{CI}): 1.118-1.551$ and

Table 3. The parameters associated with serum Elabela measurements

\begin{tabular}{|c|c|c|c|c|}
\hline & \multicolumn{2}{|c|}{$\begin{array}{l}\text { Univariate } \\
\text { analysis }\end{array}$} & \multicolumn{2}{|c|}{$\begin{array}{l}\text { Multivariate } \\
\text { analysis }\end{array}$} \\
\hline & $\mathbf{p}$ & $\mathbf{r}$ & $\mathbf{p}$ & $\beta$ \\
\hline Blood urea nitrogen & 0.023 & 0.179 & 0.960 & 0.011 \\
\hline NT-proBNP & $<0.001$ & 0.669 & 0.002 & 0.253 \\
\hline Hs-CRP & 0.038 & 0.163 & 0.944 & 0.081 \\
\hline AST (u/L) & 0.015 & 0.192 & 0.572 & 0.027 \\
\hline ALT (u/L) & 0.016 & 0.189 & 0.219 & 0.056 \\
\hline LVd dimension & $<0.001$ & 0.758 & 0.375 & 0.093 \\
\hline LVs dimension & $<0.001$ & 0.760 & 0.083 & 0.175 \\
\hline LVd volume & $<0.001$ & 0.682 & 0.401 & 0.068 \\
\hline LVs volume & $<0.001$ & 0.767 & 0.483 & 0.074 \\
\hline LVEF & $<0.001$ & -0.821 & $<0.001$ & -0.617 \\
\hline LAd dimension & $<0.001$ & 0.558 & 0.001 & 0.267 \\
\hline \multicolumn{5}{|c|}{$\begin{array}{l}\text { ALT: Alanine aminotransferase, AST: Aspartate aminotransferase, Hs- } \\
C R P \text { : High-sensitive C-reactive protein, } L A d \text { : Left atrium diastolic, LVd: } \\
\text { Left ventricular diastolic, LVEF: Left ventricular ejection fraction, } L V s \text { s: } L \text { eft } \\
\text { ventricular systolic, NT-proBNP: } N \text {-terminal brain natriuretic peptide } \\
R^{2}{ }_{\text {adjusted }}=0.686 \text { in multivariate analysis }\end{array}$} \\
\hline
\end{tabular}

$\mathrm{p}<0.001$; OR: $0.806,95 \%$ CI: $0.710-0.916$ and $p=0.001$, respectively]. According to this analysis, the increase of 1 $\mathrm{ng} / \mathrm{mL}$ increased the risk of being class III by $31.7 \%$.

\section{Discussion}

The most important finding of our study is that the serum Elabela value is significantly increased in patients with HFrEF compared to healthy controls. Elabela value was found to be significantly increased from NYHA class I to III, and the highest Elabela value was found in class III patients. In addition, we found a moderate relationship between serum Elabela level and NT-proBNP level, LVEF and LA end-diastolic diameter. Increased serum Elabela level in patients with HFrEF is moderately related to both anatomic remodeling parameters and a peptide that is sensitive and specific for HF; the Apelinergic system is thought to be a very important system in patients with HFrEF. Therefore, the data could be a contribution to the literature.

Some different effects of Apelin and Elabela over the cardiovascular system have been shown in studies. They contribute to the formation of heart and angiogenesis in the embryonic period, have inotropic effects, can cause vasodilatation in both systemic and pulmonary vascular systems, can reduce the development of cardiac hypertrophy and fibrosis, and may improve HF and myocardial infarction clinic $^{(2,4,8,13,16)}$. Therefore, it is thought that this may be a treatment method because of all these positive and cardiovascular protective effects ${ }^{(8,17)}$. In a previous study, it was shown that the serum Apelin level

Table 4. Clinic, demographic and laboratory findings of HF patients according to NYHA class (only different values between NYHA classes are shown)

\begin{tabular}{|c|c|c|c|c|}
\hline Variable & $\begin{array}{l}\text { NYHA class I } \\
(n=21)\end{array}$ & $\begin{array}{l}\text { NYHA class II } \\
(n=30)\end{array}$ & $\begin{array}{l}\text { NYHA class III } \\
(n=38)\end{array}$ & $\mathbf{p}$ \\
\hline NT-proBNP (pg/mL) & $1024 \pm 720^{\beta}$ & $1436 \pm 864$ & $1684 \pm 820$ & 0.014 \\
\hline Elabela (ng/mL) & $9.88 \pm 2.42^{\beta}$ & $11.6 \pm 3.33$ & $13.0 \pm 3.26$ & 0.002 \\
\hline Left ventricular diastolic dimension (mm) & $63.2 \pm 2.48^{\beta}$ & $65.6 \pm 5.03$ & $67.8 \pm 7.74$ & 0.022 \\
\hline Left ventricular diastolic volume (mm) & $139 \pm 14 \beta$ & $140 \pm 19$ & $152 \pm 28$ & 0.040 \\
\hline Left ventricular ejection fraction (\%) & $30.7 \pm 4.01^{\beta}$ & $29.6 \pm 3.43$ & $27.3 \pm 4.86$ & 0.007 \\
\hline Left atrium diastolic dimension (mm) & $38.9 \pm 6.01^{\beta}$ & $40.7 \pm 5.23$ & $43.4 \pm 6.08$ & 0.014 \\
\hline
\end{tabular}


increased the intracellular calcium level in patients with $\mathrm{HFrEF}^{(4,13)}$. Apelin was applied in 18 patients with NYHA class II-III HFrEF for the first time in 2010 and it can be used as a treatment in acute and chronic HF patients with its positive effects ${ }^{(8)}$. Elabela has been tested only on mice and has similar positive effects on the prevention of HF development ${ }^{(16)}$. It was first shown in 2003 that serum Apelin levels increased in patients with HFrEF. In this study, it was reported that serum Apelin level was high in the early period of the patients and serum Apelin level was decreased in the later stages ${ }^{(13)}$. Serum Apelin levels were found to be $3.58 \pm 0.33 \mathrm{ng} / \mathrm{mL}, 4.94 \pm 0.85 \mathrm{ng} /$ $\mathrm{mL}, 6.22 \pm 0.63 \mathrm{ng} / \mathrm{mL}$, and $4.58 \pm 0.62 \mathrm{ng} / \mathrm{mL}$ in patients with NYHA stage I, NYHA stage II and NYHA stage III, respectively. Similarly, serum Apelin levels were found to be in patients with normal, mild-to-moderate impaired LVEF and severe impaired LVEF were reported as $3.98 \pm 0.34 \mathrm{ng} / \mathrm{mL}, 6.02 \pm 0.72 \mathrm{ng} / \mathrm{mL}$ and $4.11 \pm 0.58 \mathrm{ng} /$ $\mathrm{mL}$, respectively ${ }^{(13)}$. As far as we have investigated, there are no clear data on serum Elabela level in patients with HFrEF. Although Elabela level was studied in our study, it was found that serum Elabela level was significantly higher in patients with HFrEF than in healthy controls in accordance with the previous study, also there was a moderate relationship between serum Elabela level and LVEF. In our study, Elabela value was found to be significantly increased from NYHA class I to III and the highest Elabela value was found in class III patient. When patients with HFrEF were separated into advanced class HF (NYHA class III) and non-advanced class HF (NYHA class I-II), the Elabela levels were found to be $10.9 \pm 3.07$ $\mathrm{ng} / \mathrm{mL}$ and $13.1 \pm 3.26 \mathrm{ng} / \mathrm{mL}$, respectively.

It is not easy to explain the pathogenesis of HF with a mechanism covering all clinical conditions. The most common form of HF is pump failure due to myocardial contractile disorder. Cardiac adaptation mechanisms aim to maintain LV stroke volume within normal limits. Chronic pressure loading is first compensated by hypertrophy. Decompensation starts when cardiac dilatation occurs after remodeling. Non-cardiac adaptation mechanisms alter the intravascular volume and vascular resistance by many different mechanisms such as the SNS, RAA system, natriuretic peptides, arginine vasopressin, prostaglandins, nitric oxide, and cytokines. These physiopathological processes, which initially increase ventricular performance, may result in decreased cardiac performance and HF symptoms occurred. Although there is no clear relationship between all these physiopathological processes and the apelinergic system, the Apelin seems to be increased for protection purposes especially after myocardial infarction remodeling phase and in HF, and to have positive effects by blocking the RAA system ${ }^{(2,4,8,13)}$. Recently, in a study conducted on mice, Elabela has been shown to protect the heart from cardiac dysfunction, hypertrophy and fibrosis development as a result of pressure over the heart ${ }^{(16)}$. To the best of our knowledge, the association between natriuretic peptides and Apelin or Elabela is unknown. Both of these apelinergic system peptides have attenuating or slowing effects over myocardial hypertrophy and fibrotic processes ${ }^{(2)}$. We feel that due to these protective effects, Elabela might have a treatment potential in some cardiovascular diseases including $\mathrm{HF}^{(17)}$. In our study, we found a moderate relationship between NT-proBNP LVEF and LA end-diastolic diameter and Elabela level in patients with HFrEF. This finding has suggested that the natriuretic and apelinergic system may be activated together with the aim of compensation in patients with HFrEF. Elabela levels might be increased to decrease LA, LV diameters and NT-proBNP levels and to increase the LVEF levels. However, this finding should be demonstrated physiopathologically.

Our primary objective was to determine the Elabela level in HFrEF patients and to compare it with healthy subjects. Nonetheless, we found that Elabela levels increased in HFrEF and found even higher Elabela levels with the advancing NYHA class. Elabela level had similar statistical significance to NT-proBNP in the prediction of NYHA class III patients in our study. Therefore, Elabela might be a new biomarker in worsened HF situations. 


\section{Study Limitations}

This study consisted of a relatively limited number of patients, and results should therefore be interpreted with caution. Only NYHA stage I-II-III patients were included in our study, but NYHA class IV patients were excluded. Therefore, patients with advanced HFrEF may also need to be included in the study. Although biochemical measurements were performed in our study, there was no study on the APJ receptor level in tissue samples. It could be more meaningful to look at myositis for similar findings. There is a close relationship between the apelinergic system and the RAA system. Although there was a correlation between the Elabela level and NTproBNP level, no biochemical analysis was performed for the RAA system. A more meaningful result could be obtained if the biochemical evaluation of the RAA system was performed. New studies should be conducted in which the RAA system and tissue samples are evaluated and patients with NYHA class IV are included. All patients who were included in the study were receiving optimal medical treatment according to their clinical findings and NYHA class. For this reason, the effect of the current treatment on the Elabela level was not evaluated. Both Elabela and Apelin are not practical clinical assessment arguments in the diagnostic or follow-up phases of HFrEF patients yet.

\section{Conclusion}

The serum Elabela level in patients with HFrEF is significantly increased, and this is moderately related to NT-proBNP, LA diastolic diameter and LVEF. Increased Elabela value in patients with HFrEF is related to the advanced class of HFrEF. However, we concluded that these results should be strengthened or supported by new studies with different and more HFrEF patients, possibly in multicenter studies. When our study and previous studies of Elabela and similar peptides were evaluated together, an increase in RAA system, natriuretic peptide and SNS, as well as an increase in the activity of the apelinergic system, was considered in patients with HF for cardiovascular protection. In particular, the association between NT-proBNP and Elabela suggests that these peptides may be activated for protection against adverse symptoms, signs and pathophysiologic conditions in patients with HF.

\section{Ethics}

Ethics Committee Approval: The study was conducted according to the recommendations of the Declaration of Helsinki and Çukurova University Faculty of Medicine, Ethical Committee of Non-invasive Clinical Research approved the protocol (decision no: 30 date: 05.10.2018).

Informed Consent: Consent forms were explained in detail to all patients and patients were included in the study after written informed consent was obtained.

Peer-review: Externally peer-reviewed.

\section{Authorship Contributions}

Concept: A.B., Y.D., Design: A.B., Y.D., Data Collection or Processing: A.B., A.A., Y.D., H.E.S., M.K., Analysis or Interpretation: Y.D., M.K., Literature Search: A.B., A.A., Y.D., H.E.S., M.K., Writing: A.B., A.A., Y.D., H.E.S., M.K.

Conflict of Interest: There is no conflict of interest with the authors and the results of the study.

Financial Disclosure: The authors declared that this study received no financial support.

\section{References}

1. Ponikowski P, Voors AA, Anker SD, et al. 2016 ESC Guidelines for the diagnosis and treatment of acute and chronic heart failure: The Task Force for the diagnosis and treatment of acute and chronic heart failure of the European Society of Cardiology (ESC) Developed with the special contribution of the Heart Failure Association (HFA) of the ESC. Eur Heart J 2016; 37: 2129-200.

2. Zhang Y, Wang Y, Lou Y, et al. Elabela, a newly discovered APJ ligand: Similarities and differences with Apelin. Peptides 2018;109:23-32.

3. Simpkin JC, Yellon DM, Davidson SM, et al. Apelin-13 and apelin-36 exhibit direct cardioprotective activity against ischemia-reperfusion injury. Basic Res Cardiol 2007;102:518-28

4. Dai T, Ramirez-Correa G, Gao WD. Apelin increases contractility in failing cardiac muscle. Eur J Pharmacol 2006;553:222-8. 
5. Murza A, Besserer-Offroy É, Côté J, et al. C-Terminal modifications of apelin-13 significantly change ligand binding, receptor signaling, and hypotensive action. J Med Chem 2015;58:2431-40.

6. Yang P, Read C, Kuc RE, et al. Elabela/Toddler is an endogenous agonist of the apelin APJ receptor in the adult cardiovascular system, and exogenous administration of the peptide compensates for the downregulation of its expression in pulmonary arterial hypertension. Circulation 2017;135:116073.

7. Murza A, Sainsily X, Coquerel D, et al. Discovery and structure-activity relationship of a bioactive fragment of ELABELA that modulates vascular and cardiac functions. J Med Chem 2016;59:2962-72.

8. Japp AG, Cruden NL, Barnes G, et al. Acute cardiovascular effects of apelin in humans: potential role in patients with chronic heart failure. Circulation 2010;121:1818-27.

9. Perjés Á, Kilpiö T, Ulvila J, et al. Characterization of apela, a novel endogenous ligand of apelin receptor, in the adult heart. Basic Res Cardiol 2016;111:2

10. Zhong JC, Yu XY, Huang Y, et al. Apelin modulates aortic vascular tone via endothelial nitric oxide synthase phosphorylation pathway in diabetic mice. Cardiovasc Res 2007;74: 388-95.

11. Zeng H, He X, Hou X, et al. Apelin gene therapy increases myocardial vascular density and ameliorates diabetic cardiomyopathy via upregulation of sirtuin 3. Am J Physiol Heart Circ Physiol 2014;306:H585-97.
12. Barnes GD, Alam S, Carter G, et al. Sustained cardiovascular actions of APJ agonism during renin-angiotensin system activation and in patients with heart failure. Circ Heart Fail 2013;6:482-91.

13. Chen MM, Ashley EA, Deng DX, et al. Novel role for the potent endogenous inotrope apelin in human cardiac dysfunction. Circulation 2003;108:1432-9.

14. Lang RM, Bierig M, Devereux RB, et al. Chamber Quantification Writing Group. Recommendations for chamber quantification: A report from the American Society of Echocardiography's Guidelines and Standards Committee and The Chamber Quantification Writing Group, developed in conjunction with the European Association of Echocardiography, a branch of the European Society of Cardiology. J Am Soc Echocardiogr 2005; 18:1440-63.

15. Schiller NB, Shah PM, Crawford M, et al. Recommendations for quantitation of the left ventricle by two-dimensional echocardiography. American Society of Echocardiography Committee on Standards, Subcommittee on Quantitation of Two-Dimensional Echocardiograms. J Am Soc Echocardiogr 1989;2:358-67.

16. Sato T, Sato C, Kadowaki A, et al. ELABELA-APJ axis protects from pressure overload heart failure and angiotensin II-induced cardiac damage. Cardiovasc Res 2017;113:760-9.

17. Yang P, Maguire JJ, Davenport AP. Apelin, Elabela/Toddler, and biased agonists as novel therapeutic agents in the cardiovascular system. Trends Pharmacol Sci 2015;36:560-7. 